BULL. AUSTRAL. MATH. SOC.

VOL. $29(1984), 377-387$.

\title{
OSCILLATIONS IN LINEAR SYSTEMS OF DIFFERENTIAL-DIFFERENCE EQUATIONS
}

\author{
K. Gopalsamy
}

\begin{abstract}
Sufficient conditions are derived for all bounded solutions of a class of linear systems of differential difference equations to be oscillatory.
\end{abstract}

\section{Introduction}

Oscillatory solutions of scalar ordinary differential equations with and without deviating arguments have been vigorously investigated by numerous authors. For a survey of results concerned with oscillations in scalar differential equations we refer to Kartsatos [4]. However to the best of the author's knowledge, oscillations in systems of (non-scalar) differential equations with or without deviating arguments have not been investigated in any detail and it appears that there is no published work on such oscillatory systems. In the following we obtain a set of sufficient conditions for all bounded solutions of a linear system of differential-difference equations of first order to be oscillatory (defined below) when the system has a single delay as well as several delays. We shall first consider systems of the form

(1.1) $\frac{d x_{i}(t)}{d t}=\sum_{j=1}^{n} a_{i j} x_{j}(t-\tau), t>0, i=1,2, \ldots, n$, where $a_{i j}$ and $\tau$ are real constants with $\tau>0$. If we denote the

Received 19 December 1983.

Copyright Clearance Centre, Inc. Serial-fee code: 0004-9727/84 $\$$ A2.00 + 0.00 . 
column vector $\left\{x_{1}(t), \ldots, x_{n}(t)\right\}^{T}$ by $\tilde{x}(t)$, then we can rewrite (1.1) in vector matrix notation as follows:

$$
\frac{d \tilde{x}(t)}{d t}=A \tilde{x}(t-\tau), t>0
$$

where $A$ denotes the $n \times n$ matrix of constants $\left\{a_{i j}, i, j=1,2, \ldots, n\right\}$. If (1.2) is supplemented with initial conditions of the form

$$
\tilde{x}(s)=\tilde{\varphi}(s)=\left\{\varphi_{I}(s), \ldots, \varphi_{n}(s)\right\}^{T}, s \in[-\tau, 0],
$$

where $\tilde{\varphi}:[-\tau, 0] \rightarrow \mathbb{R}^{n}, \tilde{\varphi}$ is continuous then one can show that solutions of $(1.2)-(1.3)$ exist on $[0, \infty)$; in fact we have, from $(1.2)-(1.3)$,

$$
\begin{aligned}
\tilde{x}(t) & =\tilde{\varphi}(0)+A \int_{0}^{t} \tilde{x}(s-\tau) d s, t>0, \\
& =\tilde{\varphi}(0)+A \int_{-s}^{t-\tau} \tilde{x}(\eta) d \eta
\end{aligned}
$$

and hence

$$
\|\tilde{x}(t)\| \leq\|\tilde{\varphi}(0)\|+\|A\| \int_{-s}^{0}\|\tilde{\varphi}(n)\| d n+\|A\| \int_{0}^{t}\|\tilde{x}(n)\| d n
$$

from which by Gronwall's inequality it will follow that

$$
\|\tilde{x}(t)\| \leq\left[\|\tilde{\varphi}(0)\|+\|A\| \int_{-s}^{0}\|\tilde{\varphi}(\eta)\| d \eta\right] \exp [\|A\| t]
$$

showing that solutions of $(1.2)-(1.3)$ are of exponential order. Thus one can use methods of Laplace transform for the study of equations of the form $(1.2)$.

Since the literature on oscillations of nonscalar systems of ordinary or delay-differential equations is (almost) nonexistent, we will adopt the following definition.

DEFINITION. A nontrivial vector $x(t)=\left\{x_{1}(t), \ldots, x_{n}(t)\right\}^{T}$ defined on $[0, \infty]$ is said to be oscillatory if and only if at least one component 
of $\tilde{x}$ has arbitrarily large zeros on $[0, \infty)$. The vector $\tilde{x}$ is said to stay away from the origin in $\mathbb{R}^{n}$ asymptotically if and only if $\underset{t \rightarrow \infty}{\lim \inf }\|\tilde{x}(t)\|=\underset{t \rightarrow \infty}{\lim \inf } \sum_{i=1}^{n}\left|x_{i}(t)\right|>0$.

We remark that the above definition of oscillatory solution vectors of nonscalar systems is not the only possible generalisation of the corresponding notion of scalar equations; however our definition of oscillatory vectors reduces to that of the familiar scalar functions on $[0, \infty)$ if the vector has trivially one component only.

\section{Oscillatory solutions}

Let $\tilde{X}(\lambda)$ denote the Laplace transform of a solution vector $\tilde{x}(t)$ of $(1.1),(1.3)$ defined by

$$
\begin{aligned}
\tilde{X}(\lambda) & =\left\{x_{1}(\lambda), \ldots, x_{n}(\lambda)\right\}^{T}, \\
X_{j}(\lambda) & =\int_{0}^{\infty} x_{j}(t) e^{-\lambda \tau} d t .
\end{aligned}
$$

It will follow from elementary properties of Laplace transforms that

$$
\begin{aligned}
\tilde{X}(\lambda) & =\left[\lambda I-A e^{-\lambda t_{-}}\right]^{-I}\left[\tilde{\varphi}(0)+A \int_{-\tau}^{0} \tilde{\varphi}(s) d s\right] \\
& =\operatorname{adj}\left[\lambda I-A e^{-\lambda \tau}\right]\left[\tilde{\varphi}(0)+A \int_{-\tau}^{0} \tilde{\varphi}(s) d s\right] / H(\lambda)
\end{aligned}
$$

where $I$ denotes the $n \times n$ identity matrix and $H(\lambda)$ is defined by

$$
H(\lambda)=\operatorname{det}\left[\lambda I-A e^{-\lambda \tau}\right]
$$

By the inversion theorem on Laplace transforms we have from (2.1)-(2.3) that any solution of $(1.1)$ is given by the integral representation,

(2.4) $\tilde{x}(t)=\frac{1}{2 \pi i} \int_{\sigma-i \infty}^{\sigma+i \infty}\left(e^{\lambda t} \operatorname{adj}\left[\lambda I-A e^{-\lambda \tau}\right]\left[\tilde{\varphi}(0)+A \int_{-\tau}^{0} \tilde{\varphi}(s) d s\right] / H(\lambda)\right) d \lambda$

where $\sigma$ is any real number greater than the real parts of roots of $H(\lambda)=0$; the existence of such a real number $\sigma$ is well known (Hale [3]). The integral in (2.4) can be evaluated using residue calculus so 
that

$$
\tilde{x}(t)=\sum_{j} \tilde{p}_{j}(t) e^{\lambda_{j} t}, t>0,
$$

where the polynomial (in $t$ ) vector $\tilde{p}_{j}(t)$ is determined by

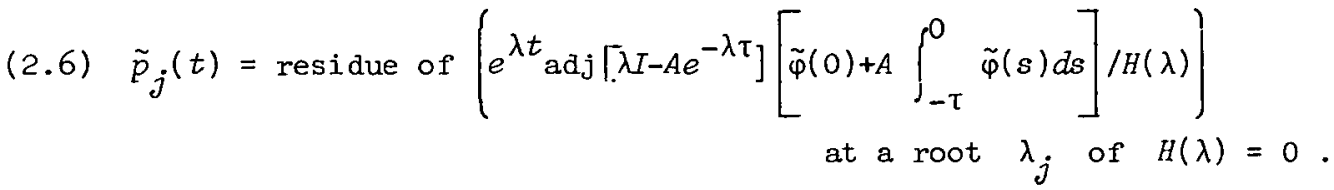

The convergence of the series representation of the type in (2.5) has been established by Banks and Manitius [1]. With these preparations we can now formulate our first result.

THEOREM 2.1. Suppose that the matmix A of real coefficients $a_{i j}$ $(i, j=1,2, \ldots, n)$ in $(1.1)$ is such that

\section{(2.7) (i) $\operatorname{det} A \neq 0$,}

(ii) the eigenvalues $\alpha_{1}, \alpha_{2}, \ldots, \alpha_{n}$ (real or complex) satisfy

$$
\left|\alpha_{j}\right| \tau>1, j=1,2, \ldots, n .
$$

Then all bounded solutions of (1.1) are oscillatory.

Proof. Since solutions of (1.1) are representable as in (2.5), it will follow that a necessary and sufficient condition for all bounded solutions of (1.1) to be oscillatory is that the characteristic equation $H(\lambda)=0$ has no real nonpositive root. Since $\alpha_{1}, \alpha_{2}, \ldots, \alpha_{n}$ are the eigenvalues of $A$, we have immediately that

$$
\operatorname{det}\left[\lambda I-A e^{-\lambda \tau}\right]=0 \Leftrightarrow \prod_{j=1}^{n}\left[\lambda-\alpha_{j} e^{-\lambda \tau}\right]=0 .
$$

Thus we are led to an investigation of the nature of the roots of

$$
\lambda=\alpha_{j} e^{-\lambda \tau}, j=1,2, \ldots, n .
$$

Suppose now that there exists a bounded nonoscillatory solution of (1.1); that is there exists a real nonpositive root say $\lambda^{*}$ such that 
(2.11) $\quad \lambda^{*}=\alpha_{j} e^{-\lambda^{*} \tau}$ for some $j \in\{1,2, \ldots, n\}$.

Since $\operatorname{det} A \neq 0, \quad \alpha_{j} \neq 0 \quad(j=1,2, \ldots, n)$, and hence $\lambda^{*} \neq 0 ;$ thus $\lambda^{*}<0$. It will then follow from (2.11) that

$$
\begin{aligned}
1 & =\tau\left|\alpha_{j}\right|\left(e^{\left|\lambda^{*}\right| \tau} /\left|\lambda^{*}\right| \tau\right) \text { for } j \in\{1,2, \ldots, n\} \\
& \geq \tau\left|\alpha_{j}\right| e \text { for some } j \in\{1,2, \ldots, n\} .
\end{aligned}
$$

But (2.12) contradicts (2.8) and hence (1.1) cannot have a bounded nonoscillatory solution when (2.7)-(2.8) hold and the proof is complete.

Let us now consider a linear delay-differential system of the form

$$
\frac{d \tilde{x}(t)}{d t}=B \tilde{x}(t)+A \tilde{x}(t-\tau), t>0
$$

where $A$ and $B$ denote real constant $n \times n$ matrices with elements $a_{i j}$, $b_{i j}(i, j=1,2, \ldots, n)$ respectively and $\tau>0$ is a constant. We will adopt the following norms of vectors and matrices:

$$
\begin{aligned}
\|\tilde{x}(t)\| & =\sum_{i=1}^{n}\left|x_{i}(t)\right|, \\
\|A\| & =\max _{j} \sum_{i=1}^{n}\left|a_{i j}\right|, \quad\|B\|=\max _{j} \sum_{i=1}^{n}\left|b_{i j}\right| .
\end{aligned}
$$

The measure $\mu(B)$ of the matrix $B$ is defined by

$$
\mu(B)=\lim _{\theta \rightarrow 0+} \frac{\|I+\theta B\|-1}{\theta}
$$

which for the chosen norms reduces to

$$
\mu(B)=\max _{j}\left[b_{j j}+\sum_{\substack{i=1 \\ i \neq j}}^{n}\left|b_{i j}\right|\right] .
$$

(For more details of the measure of a matrix we refer to Vidyasagar [5].)

THEOREM 2.2. Assume the following for the system (2.13):

(i) $\operatorname{det} A \neq 0$,

(2.14) (ii) $\mu(B)+\|A\| \neq 0$, 


\section{(2.15) (iii) $\quad(\|A\| \tau e) \exp [-\tau|\mu(B)|]>1$.}

Then all bownded solutions of (2.13) corresponding to continuous initial conditions on $[-\tau, 0]$ are oscillatory on $[0, \infty)$.

Proof. Let us rewrite (2.13) in component form so that

$$
\frac{d x_{i}(t)}{d t}=\sum_{j=1}^{n} b_{i j} x_{j}(t)+\sum_{j=1}^{n} a_{i j} x_{j}(t-\tau)
$$

and suppose that there exists a solution say $y(t)=\left\{y_{1}(t), \ldots, y_{n}(t)\right\}^{T}$ of (2.15) which is bounded and nonoscillatory on $[0, \infty)$. It will then follow that there exists a $t^{*}>0$ such that no component of $\tilde{y}(t)$ has a zero for $t>t^{*}+\tau$ and as a consequence we will have

$$
\text { (2.17) } \frac{d\left|y_{i}(t)\right|}{d t} \leq b_{i i}\left|y_{i}(t)\right|+\sum_{\substack{j=1 \\ j \neq i}}^{n}\left|b_{i j}\right|\left|y_{j}(t)\right|+\sum_{j=1}^{n}\left|a_{i j}\right|\left|y_{j}(t-\tau)\right|
$$

$$
\text { for } t \geq t^{*}+2 \tau
$$

and hence

$$
\frac{d u(t)}{d t} \leq \mu(B) u(t)+\|A\| u(t-\tau), \quad t \geq t^{*}+2 \tau,
$$

where $u(t) \equiv \sum_{i=1}^{n}\left|y_{i}(t)\right|$ and by the above preparation $u(t)>0$ for $t \geq t^{*}+\tau$. Consider now the scalar delay differential equation

$$
\frac{d v(t)}{d t}=\mu(B) v(t)+\|A\| v(t-\tau), \quad t \geq t^{*}+2 \tau
$$

with $v(s)=u(s), s \in\left[t^{*}, t^{*}+\tau\right]$. It is an elementary exercise now to show that

$$
u(t) \leq v(t) \text { for } t \geq t^{*}+2 \tau \text {. }
$$

We now claim that all bounded solutions of (2.19) are oscillatory on $\left[t^{*}+2 \tau, \infty\right)$; suppose this is not the case; then the characteristic equation associated with (2.19) given by

$$
\lambda=\mu(B)+\|A\| e^{-\lambda \tau}
$$

will have a real nonpositive root say $\lambda^{* *}$. It will follow from (2.14), $\lambda^{* *} \neq 0$. Thus $\lambda^{* *}<0$ and hence we have from 


$$
\left|\lambda^{* *}\right| \geq\|A\| e^{\left|\lambda^{* *}\right| \tau}-|\mu(B)|
$$

that

$$
\left|\lambda^{* *}\right|+|\mu(B)| \geq\|A\| e^{\left|\lambda^{* *}\right| \tau}
$$

that is,

$$
\begin{aligned}
I & \geq\left(\|A\| e^{\left|\lambda^{* *}\right| \tau}\right) /\left\{\left|\lambda^{* *}\right|+|\mu(B)|\right\} \\
& \geq\left\{\|A\| e^{-|\mu(B)| \tau} \tau\right\}\left\{\exp \left|\left(\left|\lambda^{* *}\right|+|\mu(B)|\right) \tau\right|\right\} /\left[\left\{\left|\lambda^{* *}\right|+|\mu(B)|\right\} \tau\right] \\
& \geq\|A\| \tau e e^{-\tau|\mu(B)|}
\end{aligned}
$$

and the last inequality contradicts (2.15) and hence our claim regarding the oscillatory nature of $v$ on $[0, \infty)$ is valid; since $v$ has arbitrarily larger zeros, $u$ will have arbitrarily large zeros which means that $\sum_{i=1}^{n}\left|y_{i}(t)\right|$ is oscillatory implying that $\tilde{y}(t)$ is oscillatory; but this is absurd since $\tilde{y}$ is a nonoscillatory vector. Thus there cannot exist a bounded nonoscillatory solution of (2.16) when the conditions of the theorem hold and the proof is complete.

The following result deals with oscillations in linear systems of equations with a multiplicity of delays.

THEOREM 2.3. Let $a_{i j}, \tau_{i j}(i, j=1,2, \ldots, n)$ denote real constants such that $a_{i i} \neq 0, \tau_{i i}>0(i=1,2, \ldots, n)$ and $\tau_{i j} \geq 0$ $(i, j=1,2, \ldots, n, i \neq j)$ and consider the system

$$
\frac{d x_{i \cdot}(t)}{d t}=\sum_{j=1}^{n} a_{i j} x_{i}\left(t-\tau_{i j}\right), t>0
$$

If

$$
\text { (i) } \operatorname{det} A=\operatorname{det}\left(a_{i j}\right) \neq 0 \text {, }
$$

(2.25). (ii) $\left|a_{i i}\right| \tau_{i i} e>1+e \sum_{\substack{j=1 \\ j \neq i}}^{n}\left|a_{i j}\right| \tau_{i j}$,

then all bounded solutions of (2.24) corresponding to continuous initial conditions defined on $[-\tau, 0], \tau=\max _{1 \leq i, j \leq n} \tau_{i j}$ are oscillatory on 
$[0, \infty)$.

Proof. The characteristic equation corresponding to (2.24) is given by

$$
\operatorname{det}\left[\lambda I-a_{i j} e^{-\lambda \tau_{i j}}\right]=0 .
$$

Suppose (2.24) has a bounded nonoscillatory solution. Then (2.26) has a real nonpositive root say $\delta$ such that

$$
\operatorname{det}\left[\delta I-a_{i j} e^{-\delta \tau} i j\right]=0 .
$$

Since $\operatorname{det}\left(a_{i j}\right) \neq 0, \delta \neq 0$ and hence $\delta<0$ is an eigenvalue of the matrix with entries $a_{i j} \exp \left\{-\delta \tau_{i j}\right\} \quad(i, j=1,2, \ldots, n)$. By Gershgorin's theorem (Franklin [2]), $\delta$ satisfies (2.27) $\left|\delta-a_{i i} e^{-\delta \tau} i i\right| \leq \sum_{\substack{j=1 \\ j \neq i}}^{n}\left|a_{i j}\right| e^{-\delta \tau} i j$ for some $i \in\{1,2, \ldots, n\}$. It will then follow from

$$
\begin{aligned}
& |\delta|=\left|a_{i i} e^{-\delta \tau_{i i}}+\delta-a_{i i} e^{-\delta \tau_{i i}}\right| \\
& \geq\left|a_{i i}\right| e^{-\delta \tau} i i \\
& \geq\left|a_{i i}\right| e^{\tau_{i i}|\delta|}-\sum_{\substack{j=1 \\
j \neq i}}^{n}\left|a_{i j}\right| e^{\tau_{i j}|\delta|}
\end{aligned}
$$

that

$$
|\delta|+\sum_{\substack{j=1 \\ j \neq i}}^{n}\left|a_{i j}\right| e^{\tau_{i j}|\delta|} \geq\left|a_{i i}\right| e^{\tau_{i i}|\delta|}
$$

and hence

$$
\inf _{|\delta|>0}\left\{I+\sum_{\substack{j=1 \\ j \equiv i}}^{n} \tau_{i j}\left|a_{i j}\right|\left(\frac{e^{|\delta| \tau_{i j}}}{|\delta| \tau_{i j}}\right)\right\} \geq \inf _{|\delta|>0}\left\{\left|a_{i i}\right| \tau_{i i}\left(\frac{e^{|\delta| \tau_{i j}}}{|\delta| \tau_{i i}}\right)\right\}
$$

which leads to 
(2.28)

$$
\left(1+e \sum_{\substack{j=1 \\ j \neq i}}^{n} \tau_{i j}\left|a_{i j}\right|\right) \geq\left|a_{i i}\right| \tau_{i i} e \text { for some } i \in\{1,2, \ldots, n\} .
$$

But (2.28) contradicts (2.25). Thus (2.24) cannot have a bounded nonoscillatory solution when the conditions of the theorem hold.

The following corollaries are of some interest by themselves.

COROLLARY 2.1. Suppose that the coefficient matmix $A=\left(a_{i j}\right)$ in (1.1) has at least one real negative eigenvalue say $\beta$ which is such that

$$
0<|\beta| \tau e \leq 1 ;
$$

then (1.I) has at least one bounded nonoscilzatory solution.

Proof. The portion of the characteristic equations of (J..l) (see (2.9)) corresponding to $\beta$ is given by

$$
\lambda=\beta e^{-\lambda \tau}
$$

which is equivalent to $\mu=|\beta| e^{\mu \tau}$ where $\mu=-\lambda$. It is easy to see that there exist positive real numbers $\mu$ such that $\mu=|\beta| e^{\mu \tau}$ when $|\beta| \tau e \leq 1$ and corresponding to such $\mu$, we will have a solution of (1.1) in the form $p_{\mu}(t) e^{-\lambda t}$ where $p_{\mu}(t)$ is a polynomial in $t$. A solution of the form $p_{\mu}(t) \exp [-\lambda t]$ is not oscillatory since $p_{\mu}$ can have only a finite number of zeros and the proof is complete.

COROLLARY 2.2. Consider a linear nonhomogeneous system of differential-difference equation of the form

$$
\frac{d \tilde{x}(t)}{d t}=A \tilde{x}(t-\tau)+\tilde{f}(t), \quad t>0,
$$

where the matrix $A$ and the constant $\tau$ are as in Theorem 2.1. Assume that $\tilde{f}:[0, \infty) \rightarrow \mathbb{R}^{n}, \tilde{f}$ is continuous on $[0, \infty)$ and $\int_{0}^{\infty}\|\tilde{f}(t)\| d t<\infty$. Then every bounded solution of (2.31) corresponding to continuous initial conditions is oscillatory on $[0, \infty)$ if such a solution stays away from the origin in $\mathbb{R}^{n}$ as $t \rightarrow \infty$.

Proof. Since $\|\tilde{f}(t)\| \rightarrow 0$ as $t \rightarrow \infty$, all the poles of the components 
of the Laplace transform of $\tilde{f}$ can have only negative real parts; with this observation, the remainder of the proof is as in that of Theorem 2.1 and we will omit the details.

\section{Nonautonomous systems}

The techniques of Laplace transform are not directly applicable in general for the study of systems with variable coefficients. However one can compare the nonautonomous system with a suitable autonomous system and derive conclusions on the behaviour of the nonautonomous systems. We briefly illustrate such a procedure in the following:

THEOREM 3.1. Let $p_{i j}:[0, \infty) \rightarrow(-\infty, \infty), p_{i j}$ $(i, j=1,2, \ldots, n)$ be bounded and continuous on $[0, \infty)$. Let $P(t)$ denote the $n \times n$ matrix with elements $p_{i j}(t), i, j=1,2, \ldots, n$. Suppose $\tau$ is a positive constant. If

$$
\left|p^{*}\right| \tau e>1
$$

where $p^{*}=\sup _{t \geq 0} \mu(P(t)), \mu(\cdot)$ denoting the matrix measure (defined earlier) then all bounded solutions of the system

$$
\frac{d x_{i}(t)}{d t}=\sum_{j=1}^{n} p_{i j}(t) x_{j}(t-\tau), t>0,
$$

corresponding to continuous initial conditions are oscillatory on $[0, \infty)$.

Proof. Suppose there exists a solution say $\tilde{y}(t)=\left\{y_{1}(t), \ldots, y_{n}(t)\right\}$ of (3.2) which is bounded and nonoscillatory. Then it will follow that there exists a $t^{*}>0$ such that for $t>t^{*}+\tau$, no component of $\tilde{y}$ has a zero. In such a case we will have

$$
\frac{d}{d t} \sum_{i=1}^{n}\left|y_{i}(t)\right| \leq \mu(P(t)) \sum_{i=1}^{n}\left|y_{i}(t-\tau)\right| \text { for } t>t^{*}+2 \tau
$$

and hence

$$
\frac{d u(t)}{d t} \leq p^{*} u(t-\tau), \quad u(t)=\sum_{i=1}^{n}\left|y_{i}(t)\right|, \quad t>t^{*}+2 \tau .
$$

A comparison of (3.3) with an equation of the form 


$$
\frac{d v(t)}{d t}=p^{*} v(t-\tau), t>t^{*}+2 \tau, v(s)=u(s), s \in\left[-\tau, t^{*}+2 \tau\right]
$$

will lead to $0<u(t) \leq v(t)$ for $t>t^{*}+2 \tau$; furthermore when (3.1) holds by Theorem 2.1 (as a special scalar case), it will follow that $v$ is oscillatory and hence $u$ is oscillatory which is a contradiction. Thus (3.2) cannot have a bounded nonoscillatory solution when (3.1) holds and the proof is complete.

We conclude with a remark that oscillations in nonlinear (nonscalar) systems of differential-difference equations remains virtually an untouched subject and there is an urgency to explore this subject matter due to its potential applications in population dynamics.

\section{References}

[1] H.T. Banks and A. Manitius, "Projection series for retarded functional differential equations with applications to optimal control problems", J. Differential Equations 18 (1975), 296-332.

[2] J.N. Franklin, Matrix theory (Prentice Hall, London, 1968).

[3] J.K. Hale, Theory of functional differential equations (SpringerVerlag, Berlin, Heidelberg, New York, 1977).

[4] A.G. Kartsatos, "Recent results on oscillation of solutions of forced and perturbed non-linear differential equations of even order",

(Lecture notes in pure and applied mathematics, 28. Marcel Dekker, New York, 1977).

[5] M. Vidyasagar, Nonlinear systems conalysis (Prentice Hall, Englewood Cliffs, New Jersey, 1978).

School of Mathematical Sciences,

Flinders University,

Bedford Park,

South Australia 5042,

Australia. 